\title{
Cross-cultural adaptation of the Management of Aggression and Violence Attitude Scale for nurse in emergency department
}

Original article

Rui Chang, Hui Yang*

Shanxi Medical University, Taiyuan, Shanxi 030001, China

Received: 24 July 2018; Accepted: 29 September 2018; Published: 20 June 2019

Abstract: Objective: To follow the guidelines of intercultural adaptation provided by the American Academy of Orthopedic Surgeons' (AAOS) Evidence-Based Medicine Committee, translating the original scale and evaluating the reliability and validity, and then to compile the Chinese version of the Management of Aggression and Violence Attitude Scale (MAVAS) for nurses in emergency room in the mainland of China.

Methods: This study consists of two phases of testing: (1) translation: forward translation, synthesis, back translation, expert committee review, and pretesting; (2) psychometric properties: content and construct validity, internal consistency, and test-retest reliability. Results: The Chinese version of MAVAS and the original version showed excellent similarities and equivalence. The average Scalelevel Content Validity Index was 0.904 , and the Item-level Content Validity Index ranged from 0.80 to 1.00 . The construct validity was tested using confirmatory factor analysis by LISREL $8.7 ; \chi^{2} / d f$ of the scale was $4.781<5$, NFI, NNFI, CFI, IFI $>0.90$, indicating that the scale's factor structure model fitted better. The internal consistency was satisfactory (scale, Cronbach's $\alpha=0.94$; subscales, Cronbach's $\alpha=0.74-0.90$ ), and the test-retest reliability over 2 weeks was good (scale, Pearson's coefficient=0.996; subscales, Pearson's coefficient $=0.801-0.963$ ).

Conclusions: The Chinese version of MAVAS had an excellent feasibility. It was found to be a valid and reliable tool to assess nurses' attitude toward patients' violence in emergency department.

Keywords: violence • cross-cultural adaptation • MAVAS • emergency department, ED • psychometric properties

(c) Shanxi Medical Periodical Press.

\section{Introduction}

Patients' aggression and violence toward health-care workers belong to a common global social security issue in various hospitals. ${ }^{1}$ According to the Report of Violent Injuries in Hospitals published by the Chinese Hospital Association, the number of violent incidents in hospitals in our country has been greatly increasing, ${ }^{2}$ and the "blowout" hospital violence put clinicians in an extremely dangerous position.

According to the International Council of Nurses (ICN), "The nurses may experience as much as 3 times more violence than any other staff." 3 The emergency department (ED) is one of the highest risk areas for patients' violence. ${ }^{4}$ Many studies show that ED nurses experience significantly more verbal violence than

How to cite this article: Chang R, Yang H. Cross-cultural adaptation of the Management of Aggression and Violence Attitude Scale for nurse in emergency department. Front Nurs. 2019; 2: xx-xx. 
physical, $74.0 \%$ of nurses have experienced physical violence every year and $88.0 \%$ have been exposed to verbal abuse..$^{5}$ American Emergency Nursing Association surveyed 6504 nurses in 2011 and found that $54.4 \%$ of nurses experienced verbal violence, of which pure language violence accounted for $42.9 \%$, language and physical violence $11.2 \%$, and physical violence only $0.8 \%{ }^{6}$ The incidence of violence suffered by nurses has increased year by year in the UK. In China, 66\% of nurses in Hong Kong ${ }^{7}$ have suffered violent injuries, $62 \%$ of nurses in Taiwan ${ }^{8}$ have experienced violent incidents, and on the mainland the incidence of violence toward nurses in the emergency health-care department is as high as $80.7 \% .^{9}$

$\mathrm{ICN}^{10}$ believes that when a nurse suffers from patient's violence, it not only affects her physical and mental health but also reduces the morale of the nurse, which leads to a decrease in the quality of care and even the loss of the whole health-care system. There is a series of adverse consequences such as job burnout and the intention of demission after nurses experienced violence, ultimately affecting the patients' well-being.

In recent years, the loss of nurses is the focus in many countries. One of the reasons may be the patient's violence. Moreover, with the statement of the Former Chairman, Li Xiuhua, the future development of nursing will surely require a large number of nurses. Therefore, it is important to reduce the harm of patients' violence for nurses, to improve nurses' working environment, and to improve nurses' satisfaction with their jobs, so as to promote the long-term development of nursing in our country.

The most widely used scale is the Management of Aggression and Violence Attitude Scale (MAVAS), which was developed by Duxbury, ${ }^{11}$ UK, in 2003 to measure the attitudes of the nurses and patients toward the causes and management on patients' aggressions and violence in mental settings. There are a total of 27 items, belonging to four dimensions, including internal causes (patients' own factors, such as disease type and disease symptoms), external causes (environmental factors) and interactions between medical staff and one dimension of management of violence. The assessment uses a visual analog scale (0-100 points), with lower scores indicating agreement and higher scores indicating disagreement. After some investigation, the scale shows good reliability and validity. In 2013, Pulsford et al. ${ }^{12}$ adapted the scale, and the adjusted scale adopted 5-point Likert scale, with 1 being very disagree and 5 being very much in agreement. In 2015, Prof. Wong ${ }^{13}$ of the Hong Kong Polytechnic University introduced the scale into the ED and compiled it into an English version. The compiled scale contains 27 items and is divided into four dimensions: internal dimension (five items), external dimension (three items), interaction dimension (five items), and management dimension (14 items). A 5-point Likert rating was used, with 1 being "strongly agree" and 5 being "strongly disagree," with lower scores indicating higher approval. Cronbach's $\alpha=0.40-0.77$, and the test-retest reliability was 0.85 . Four common factors (eigenvalues $>1$ ) were extracted from the exploratory factor analysis, and the cumulative interpretation variance was $43.4 \%$. The total Scale-level Content Validity Index $(\mathrm{S}-\mathrm{CVI})=0.97$, and the Item-level Content Validity Index $(\mathrm{I}-\mathrm{CVI})=0.80-1.00$. It has been verified that the scale can be well applied to assess the attitude of nurses in the EDs in Hong Kong for the causes and management of patients' violence.

\section{Methods}

\subsection{Translation procedures}

First, the author contacted Dr. Joy Duxbury and Prof. Wong, who are the authors of the original scale, and explained the reasons for requesting and obtained the authorization. This study followed the cross-cultural adaptation guidelines ${ }^{14}$ provided by the American Academy of Orthopedic Surgeons' (AAOS) EvidenceBased Medicine Committee and Guillemin et al.'s ${ }^{15}$ cross-cultural adaptation criterion, and according to the "forward translation, synthesis, back translation, expert committee review, pretesting" 16 translated the original scale and evaluated the reliability and validity of the revised scale so that it is basically equivalent to the original.

Stage 1: forward translation: Both the author and another nursing expert who were proficient in English, understood the project, and possessed much medical knowledge independently translated the original scale into Chinese, named $\mathrm{T} 1$ and $\mathrm{T} 2$, respectively. The researcher proofread the two drafts and recorded the words that were uncertain and controversial to form the first review report. At the same time, two English teachers whose native language is Chinese were invited to independently translate the original scale, both of them had at least 8 years of English teaching experience and neither had medical knowledge nor were not familiar with the subject background, named T3 and T4, respectively, then the drafts were proofread and recorded by the author to form the second review report.

Stage 2: synthesis: The author integrated the English original scale, four Chinese drafts, and two review reports to the team, and the members analyzed and discussed especially the controversial and uncertain 
phrases during the translation until all of them reached a consensus to form the T5 version. The author recorded all issues and solutions during the discussion.

Stage 3: back translation: To ensure consistency of content and concept between the Chinese version and the English version, the research group invited a Chinese nursing professor who lived in Canada for many years and a PhD nurse to translate the integrated T5 version into English independently and invited two English professional graduate students with no medical background also to back translate the $\mathrm{T} 5$ version so that the researcher can find out the differences in translation of the certain words and avoid the information bias.

The team members discussed on the translations of four English versions, translated and back translated repeatedly those words that were different and controversial from the original scale, and modified the expressions that did not accord with the culture and customs of the mainland in China. The Chinese translation draft T6 was formed, and the author recorded the details in the stage.

Stage 4: expert committee review: To ensure the equivalence between the Chinese version and the original scale, the author submitted the original scale, the Chinese translation of six editions, and all the recorded notes to the experts for two rounds of expert consultation (Delphi survey) to form a pilot Chinese version of the MAVAS. A total of 25 experts in this field were selected nationwide, including 13 in nursing management, two in nursing education, five in ED nurses, two in long-term research in patient violence, two in psychologists, and one public health expert, in which all of them working for at least 10 years, whose academic qualifications are master and above and deputy chief nurse and above.

The aim of the expert consultation mainly includes the following: (1) whether the language is clear; (2) whether the meaning expressed by the translation is clear; (3) whether the translation expression conforms to the mainland culture; (4) whether the content of the item is related to the measured dimensions; (5) what statistical test methods are needed in the adjustment process.

Stage 5: pretesting: This study selected 30 nurses from EDs of general hospitals in Taiyuan to conduct a questionnaire survey. The purpose of the study was to test the level of understanding of the contents of the pilot Chinese version of MAVAS, whether the expressions were clearly stated or any difficulty. In addition, the nurse marked out the items with the above conditions and corresponding amendment opinions. The author conducted semi-structured interviews with the nurses and asked questions in detail, answered questions in the filling process, and made timely feedback to experts for consultation and revision.

\subsection{Participants and settings}

A questionnaire survey was conducted among 135 emergency nurses with a convenient sampling method from six general hospitals in Taiyuan. After 2 weeks, 30 ED nurses were selected again for retesting. Inclusion criteria were as follows: (1) possess a nursing professional qualification certificate; (2) emergency nurses who voluntarily participated in this study. Exclusion criteria were as follows :(1) nurses who are transferred to and studied in the ED; (2) ED nurses on business trip or on vacation; (3) nurses working in the ED for less than 6 months; (4) unwilling to cooperate with this study; (5) nurses with serious injury during the study; (6) nurses who volunteered to withdraw from the study without completing the study.

\subsection{Ethical considerations}

This study follows the ethical principle of informed consent. First, the author contacted the head of nurses of the surveyed hospital and introduced the information of the study to them, such as the purpose of the research, significance, and research process. After obtaining their approval and cooperation, the researcher explained to the head nurse of the ED in their hospitals. The head nurse then focused on the nurses in the department, and the nurses voluntarily participated in the study. During the study, nurses may withdraw from the study at any time for various reasons and do not assume any responsibility. All surveys are anonymous and secret.

\subsection{Psychometric properties}

The reliability was assessed with internal consistency and test-retest reliability after 2 weeks. S-CVI and I-CVI were used to evaluate the content validity by 10 experts rated, and the confirmatory factor analysis (CFA) was used to test the construct validity. The survey using Excel 2010 to collect the original data was conducted; the data into the IBM SPSS 23.0 for Windows and LISREL 8.7 software for statistical analysis were recorded; and the reliability and validity of the scale were evaluated.

\section{Results}

\subsection{Translation}

Translators repeatedly checked the item that is uncertain and controversially translated and back-translated for many times until all translators reached a consensus and to make a pilot version. Then, the researchers 
completed the pilot version by Delphi survey until a formal version of the scale was made.

During the stage of Delphi survey, all experts believed that the aggression and violence of the patients are behaviors rather than a disease, they are not ethical and legal by using drugs to control the patients who are violent in the ED nor is it in line with the cultural background of mainland in China. Therefore, item 13 "Medication is a valuable approach for treating aggressive and violent behavior," item 19 "Alternatives to the use of containment and sedation to manage patient violence could be used more frequently," and item 25 "Prescribed medication should be used more frequently to help patients who are aggressive and violent" were deleted. Last but not least, experts suggested that emergency nurses should be able to identify the reasons for the patients' violence whether it is the status of mental illness or personal traits and other factors, so in the "management" dimension, all experts agreed to add the item "Nurses should conduct real-time assessments, monitoring and care of the suspicious patients who was aggression and violence." After the integration of all opinions, a second expert consultation was conducted, and in this round the result of consultation was basically consistent, and then a pilot Chinese MAVAS scale was formed finally.

\subsection{Psychometric properties 3.2.1. Sample characteristics}

One hundred and thirty-five nurses in the ED in hospitals of Taiyuan were surveyed in the study, and all of them completed the questionnaire; the response rate was $100 \%$. Table 1 summarizes the detailed data.

\subsubsection{Item analysis}

Item analysis is aimed to check the reliability and appropriateness of items of the whole scale, and the results can be used as the basis for modifying or deleting the certain items. ${ }^{17}$ In this study, the most commonly methods were used to evaluate the scale which included critical ratio (CR) method and correlation analysis.

The CR method or the extreme value method derives the degree of discrimination by calculating the "decision value" of each item. ${ }^{17}$ The higher the $C R$ value, the stronger the discrimination of the item. It is generally believed that if the $C R$ value is $<3.000$, indicating that the item is poorly identified, the item should be deleted. ${ }^{17}$ The correlation coefficient method is a kind of homogeneity test method that uses the Pearson's difference correlation to find the correlation coefficient between individual items and the total score of the scale. As we know, the item's correlation coefficient of ${ }^{3} 0.4$ is very good, $0.3-0.39$ is good, $0.2-0.29$ is fair, and $<0.2$ is bad. ${ }^{17}$ Therefore, if the score of scale item does not reach the level of significance or the correlation coefficient is $<0.3$, it means that the item and the scale are of low correlation and should be deleted (Table 2 summarizes the detailed data). The CR value of all items is between 3.334 and 12.000 , and the correlation coefficient is between 0.342 and 0.832 . All items of the scale reached a significant level and are statistically significant, indicating that no items should be deleted.

\subsubsection{Reliability}

The internal consistency was satisfactory, in which the total scale's Cronbach's $\alpha=0.938$, and the four subscales' Cronbach's $\alpha=0.740-0.900$; the test-retest reliability over 2 weeks was good, the whole scale's Pearson's coefficient $=0.996$, and the subscales' Pearson's coefficient $=0.801-0.963$.

\subsubsection{Validity}

\subsubsection{Content validity}

After two rounds of Delphi survey, 10 experts were selected to reevaluate on the validity of the scale. The S-CVI and I-CVI of the MAVAS were rated by experts in terms of the relevance of its individual items, using a 5-point Likert scale (1, irrelevant; 2 , major revision to be relevant; 3 , minor revision to be relevant; 4 , general relevant, and 5 , highly relevant). It is generally believed that when the $\mathrm{CVI}$ is $>0.8,{ }^{17}$ the content validity of the scale is good. In this study, the average S-CVI is 0.904 , and the I-CVI is $0.800-1.000$, indicating a satisfactory content validity.

\subsubsection{Construct validity}

The construct validity was assessed using CFA by LISREL 8.7. CFA is based on a specific theory to make reasonable assumptions about the relationship between latent variables and observed variables and perform statistical tests. ${ }^{18}$ The commonly used indicators are $\chi^{2} / d f, \mathrm{NFI}, \mathrm{NNFI}, \mathrm{CFI}$, and IFI (Table 3).

$\chi^{2} / d f$ indicates that the closer the value is to 1 , the better the model fit. ${ }^{19}$ Generally, the ratio is considered to be a good fit between 2 and 5 , and in fact as the sample size is large, the ratio around 5 is also be accepted, ${ }^{20,21}$ and other indicators of $>0.90$ show a good fit. ${ }^{19}$ The study shows that $\chi^{2} / d f=4.781<5, N F I=0.91$, $\mathrm{NNFI}=0.92, \mathrm{CFI}, \mathrm{IFI}=0.93$, indicating a satisfactory construct validity. 


\begin{tabular}{l}
\hline Project \\
\hline \hline Gender \\
Female \\
Male \\
Age (years) \\
$20-29$ \\
30-39 \\
40-49 \\
Educational background \\
College \\
Undergraduate \\
Master and above \\
Job title \\
Nurse \\
Primary \\
Intermediate \\
Deputy Chief \\
Job position \\
Nurse \\
Head nurse \\
Marital Status \\
Unmarried \\
Married \\
Hiring form \\
Formal establishment \\
Contract system \\
Temporary workers \\
Only child \\
Yes \\
No \\
Not
\end{tabular}

Work experience of nursing (years)

$$
\begin{aligned}
& 0-5 \\
& 6-10 \\
& 11-15 \\
& 16-20 \\
& >20
\end{aligned}
$$

Work experience in ED (years)

$$
0-5
$$$$
6-10
$$$$
11-15
$$$$
16-20
$$$$
>20
$$

Education related to management of patient violence

Yes

No

Experiences in management of patient violence

No

\section{Frequency (\%)}

$117(86.7)$

18 (13.3)

62 (45.9)

62 (45.9)

11 (8.2)

15 (11.1)

$119(88.1)$

$1(0.8)$

27 (20)

71 (52.6)

36 (26.7)

$1(0.7)$

131 (97.0)

4 (3.0)

41 (30.4)

94 (69.6)

38 (28.2)

96 (71.1)

$1(0.7)$

37 (27.4)

98 (72.6)

52 (38.5)

$41(30.4)$

18 (13.3)

16 (11.9)

8 (5.9)

62 (45.9)

35 (25.9)

21 (15.6)

$11(8.1)$

6 (4.5)

44 (32.6)

91 (67.4)

73 (54.1)

62 (45.9)

(Continued)

\begin{tabular}{lc}
\hline Project & Frequency (\%) \\
\hline \hline Interventions in managing patient violence & \\
Physical constraints & $20(14.8)$ \\
Risk assessment & $33(24.4)$ \\
De-escalation & $49(36.3)$ \\
Drug intervention & $3(2.2)$ \\
Seclusion & $6(4.4)$ \\
Others & $24(17.9)$ \\
Experienced patient violence & \\
Yes & $70(51.9)$ \\
No & $65(48.1)$ \\
History of being verbally abused in the past 6 & \\
months & \\
No & $49(36.3)$ \\
Yes & $86(63.7)$ \\
1-5 times & $61(45.2)$ \\
$6-10$ times & $13(9.6)$ \\
11-15 times & $3(2.2)$ \\
16-20 times & $0(0)$ \\
>20 times & $9(6.7)$ \\
History of being physical aggression in the past 6 & \\
months & \\
No & $122(90.4)$ \\
Yes (1-5times) & $13(9.6)$ \\
\hline & \\
\hline & \\
\hline
\end{tabular}

Table 1. General demographic data of the study $(n=135)$.

\section{Discussion}

\subsection{Translation}

In recent years, patients' violence causes a serious physical and psychological harm to nurses and is a barrier to the development of the nursing industry, which has been widespread in China. Therefore, understanding the attitude of nurses to the violence could help managers to propose targeted interventions. Our research on patients' violence is still in the initial stage in China. At present, there is no tool that can evaluate nurses' attitudes toward the violence, and there are two methods for the generation of assessment tools: ${ }^{22}$ one is the selfdesigned scales, which are time-consuming and costly; another is revising the foreign mature scales, which are used in the practice of nursing in China after crosscultural adaptation. It is relatively simple, convenient, and relatively common. MAVAS has already been introduced into other countries and regions and identified it as an excellent tool. ${ }^{23,24}$ The present study introduced MAVAS developed by Dr. Joy Duxbury, which was introduced to Hong Kong, China, by Prof. Wong of the Hong Kong Polytechnic University in 2015. However, although 


\begin{tabular}{lrc}
\hline Item & CR & Correlation coefficient \\
\hline \hline Item 1 & 5.708 & 0.570 \\
Item 2 & 6.415 & 0.571 \\
Item 3 & 4.773 & 0.696 \\
Item 4 & 5.027 & 0.516 \\
Item 5 & 4.286 & 0.459 \\
Item 6 & 8.368 & 0.685 \\
Item 7 & 8.942 & 0.757 \\
Item 8 & 10.712 & 0.808 \\
Item 9 & 10.449 & 0.756 \\
Item 10 & 8.068 & 0.667 \\
Item 11 & 7.599 & 0.732 \\
Item 12 & 10.718 & 0.825 \\
Item 13 & 8.874 & 0.739 \\
Item 14 & 12.000 & 0.832 \\
Item 15 & 6.437 & 0.633 \\
Item 16 & 6.435 & 0.717 \\
Item 17 & 3.334 & 0.342 \\
Item 18 & 10.509 & 0.762 \\
Item 19 & 7.573 & 0.689 \\
Item 20 & 5.150 & 0.512 \\
Item 21 & 7.273 & 0.650 \\
Item 22 & 8.547 & 0.641 \\
Item 23 & 4.247 & 0.437 \\
Item 24 & 6.315 & 0.363 \\
Item 25 & 5.092 & 0.580 \\
\hline & & \\
\hline
\end{tabular}

Table 2. Item analysis of the pilot MAVAS

the mainland and Hong Kong are both parts of China, the language used in the two places is different. Hong Kong still uses the English version of the scale, but mainland uses the Chinese version of the scale. Therefore, there is still a need for translation and psychological test ${ }^{25}$ of the scale, testing the reliability and validity of the Chinese version of the scale and evaluating its applicability in the mainland.

To ensure the equivalence of two versions of scales, this study selected the translators who are proficient in English including nursing graduate students and college English teachers to translate independently. In addition, a Chinese nursing professor who lived in Canada for many years, a PhD nurse, and English major graduates to back translate were selected, and 25 experts from the country in the areas of nursing management, nursing education, emergency medicine, riot prevention, psychology, and public health to discuss and reach a consensus to form a pilot Chinese version of MAVAS were invited. Then, 30 ED nurses who met the project's inclusion criteria were selected to conduct preliminary experiments. Based on the survey results and feedback,

\begin{tabular}{|c|c|c|c|}
\hline Item & Loading & Standard error & $P$ \\
\hline \multicolumn{4}{|c|}{ Internal } \\
\hline $\mathrm{A} 1$ & 0.68 & 0.07 & $<0.05$ \\
\hline A2 & 0.70 & 0.07 & $<0.05$ \\
\hline A3 & 0.81 & 0.06 & $<0.05$ \\
\hline A4 & 0.76 & 0.08 & $<0.05$ \\
\hline A5 & 0.43 & 0.08 & $<0.05$ \\
\hline \multicolumn{4}{|c|}{ External } \\
\hline A6 & 0.82 & 0.07 & $<0.05$ \\
\hline A7 & 0.98 & 0.06 & $<0.05$ \\
\hline A8 & 1.00 & 0.05 & $<0.05$ \\
\hline \multicolumn{4}{|c|}{ Interaction } \\
\hline A9 & 0.98 & 0.06 & $<0.05$ \\
\hline A10 & 0.79 & 0.06 & $<0.05$ \\
\hline A11 & 0.88 & 0.06 & $<0.05$ \\
\hline A12 & 0.98 & 0.05 & $<0.05$ \\
\hline A13 & 0.96 & 0.06 & $<0.05$ \\
\hline \multicolumn{4}{|c|}{ Management } \\
\hline A14 & 0.97 & 0.05 & $<0.05$ \\
\hline A15 & 0.85 & 0.07 & $<0.05$ \\
\hline A16 & 0.85 & 0.06 & $<0.05$ \\
\hline A17 & 0.29 & 0.07 & $<0.05$ \\
\hline A18 & 0.92 & 0.06 & $<0.05$ \\
\hline A19 & 0.75 & 0.06 & $<0.05$ \\
\hline A20 & 0.50 & 0.07 & $<0.05$ \\
\hline A21 & 0.64 & 0.06 & $<0.05$ \\
\hline A22 & 0.62 & 0.07 & $<0.05$ \\
\hline A23 & 0.35 & 0.07 & $<0.05$ \\
\hline A24 & 0.54 & 0.07 & $<0.05$ \\
\hline A25 & 0.64 & 0.06 & $<0.05$ \\
\hline
\end{tabular}

Table 3. Results of CFA of coefficient estimation.

the scale was revised in conjunction with the opinions of experts so that it accorded with the culture of the mainland.

With the scientific and rigorous cross-cultural adjustment, it was confirmed that the Chinese version of MAVAS was suitable in the mainland and maintained good equivalence with the English version of the scale.

\subsection{Psychometric properties}

It may affect the quality of the questionnaire if one takes a long time to complete it. ${ }^{19}$ In this study, the nurses actively cooperated with the survey and the average time for completing the questionnaire was about 8 minutes, and the response rate was $100 \%$, indicating a good feasibility of the scale. 
During item analysis, the CR for all items was $>3.000$ and the correlation coefficient was $>0.30$, all reached the level of statistical significance $(P>0.05)$ and should be reserved.

The internal consistency coefficient and test-retest reliability were used to evaluate the reliability of the scale. In general, Cronbach's $\alpha>0.90$, indicating excellent internal consistency reliability; $0.80-0.90$, indicating that internal consistency reliability was very good; $0.70-0.80$, which should be revised slightly but still valuable. ${ }^{17}$ The total scale's Cronbach's $\alpha=0.938$, and the four subscales' Cronbach's $\alpha=0.740-0.900$, showing the internal consistency was satisfactory. Generally, the test-retest reliability interval of 1-4 weeks was appropriate, and the test-retest reliability over 2 weeks in the research was good, the whole scale's Pearson's $r=0.996$, and the subscales' Pearson' $r=0.801-0.963$, which is higher than the results of the original scale (Pearson's $r=0.89)^{11}$ and the Hong Kong version (Pearson's $r=0.85) .{ }^{13}$

The validity of the scale was assessed using content validity and construct validity. This study invited 10 experts in various fields which included the areas of nursing management, nursing education, emergency professional, violence prevention, medical psychology, public health, and experts in statistics to evaluate the content and quality of the items. The average $\mathrm{S}-\mathrm{CVI}$ is 0.904 , and the I-CVI is $0.800-1.000$, confirming that the scale has a good content validity, and the result is consistent with the Hong Kong version. ${ }^{13}$ CFA was used to evaluate the construct validity of the

\section{References}

1. Jansen GJ, Dassen TW, Groot Jebbink G. Staff attitudes towards aggression in health care: $\overline{\text { a review }}$ of the literature. J Psychiatr Ment Health Nurs. 2005;12:3-13.

2. Jia XL, Zhou HZ, Zhao Y, et al. Investigation on hospital violence during 2003 to 2012 in China. Chin Hospitals. 2014;3:1-3.

3. Judith AO. The ICN theme in 2001: joint opposition to violence. J Chin Nurs Assoc. 2001;15:10-32.

4. Kansagra SM, Rao SR, Sullivan AF, et al. A survey of workplace violence across 65 U.S. emergency departments. Acad Emerg Med. 2008;15:68-74.

5. May DD, Grubbs LM. The extent, nature, and precipitating factors of nurse assault among three groups of registered nurses in a regional medical center. Emerg Nurs. 2002;28:1-7.

6. Emergency Nurses Association. Emergency department violence surveillance study. https:// www.ena.org/docs/default-source/resource-library/ scale. The study shows that $\chi^{2} / d f=4.781<5, \mathrm{NFI}=0.91$, $\mathrm{NNFI}=0.92, \mathrm{CFI}, \mathrm{IFI}=0.93$, indicating a satisfactory construct validity.

\section{Conclusions}

Through the cross-cultural adjustment, it has been proved that the Chinese version of the MAVAS has a good cultural adaptability, reliability and validity. The researcher fed back the revised scale to the original author and experts, and there was a widespread consensus. They all agreed that the Chinese version of the MAVAS is suitable for testing the emergency nurses' attitude toward the causes and management of patients' violence in the mainland of China.

\subsection{Limitations}

Due to the limited time and conditions, the present study investigated only the attitude of nurses in ED for the causes and management of patient violence but no patients' attitude. Therefore, in the future, it is necessary to continue to investigate the attitudes of the ED patients and their family members toward the causes and management methods of violence so that managers can formulate the specific and effective measures.

\section{Conflicts of interest}

All contributing authors declare no conflicts of interest. practice-resources/workplace-violence/2011-emergency-department-violence-surveillance-report. pdf?sfvrsn=5ad81911_4. Accessed March 11, 2018.

7. Kwok RP, Law YK, Li KE, et al. Prevalence of workplace violence against nurses in Hong Kong. Hong Kong Med J. 2006;12:6-9.

8. Lin YH, Liu HE. The impact of workplace violence on nurses in South Taiwan. Int J Nurs Stud. 2005;42:773-778.

9. Feng $X Q$, Fang $P P$, Gan $X N$, et al. Analysis on the status and influencing factors of medical violence at work in emergency departments of grade III-A general hospitals in China. Chin $J$ Emerg Med. 2017;26:337-340 (in Chinese).

10. Rego A, Sousa F, Marques C, et al. Authentic leadership promoting employees' psychological capital and creative. J Business Res. 2012;65:429-437. 
11. Duxbury J. Testing a new tool: the Management of Aggression and Violence Attitude Scale (MAVAS). Nurse Res. 2003;10:39-52.

12. Pulsford D, Crumpton A, Baker A, Wilkins T, Wright $\mathrm{K}$, Duxbury J. Aggression in a high secure hospital: staff and patient attitudes. J Psychiatr Ment Health Nurs. 2013;20:296-304.

13. Wong WK, Chien WT. Psychometric properties of the Management of Aggression and Violence Attitude Scale in Hong Kong's emergency care setting. Int Emerg Nurs. 2017;31:46-51.

14. Beaton DE, Bombardier C, Guillemin F, Ferraz MB. Guidelines for the process of cross- cultural adaptation of self-report measures. Spine (Phila Pa 1976). 2000;25:3186-3191.

15. Guillemin F, Bombardier C, Beaton D. Cross-cultural adaptation of health-related quality of life measures: literature review and proposed guidelines. $J$ Clin Epidemiol. 1993;46:1417-1432.

16. Mozzanica F, Robotti C, Ginocchio D, et al. Crosscultural adaptation and validation of the Italian Version of the Voice Symptom Scale (I-VoiSS). J Voice. 2017;31:773.e1-773.e10.

17. Wu ML. Statistical Analysis of Questionnaires SPSS Operation and Application. Chongqing: Chongqing University Press; 2010 (in Chinese).

18. He M. Study on Chinese Version of the Nurse Manager Practice Environment Scale, Evaluation and
Application. Taiyuan: Shanxi Medical University; 2014 (in Chinese).

19. Sun ZQ, Xu YY. Medical Statistics. 3rd ed. Beijing: People's Medical Publishing House; 2010 (in Chinese).

20. Qiu YZ, Lin BF. Principles and Applications of Structural Equation Models. Beijing: China Light Industry Press; 2009 (in Chinese).

21. Wu ML. Structural Equation Model: Operation and Application of AMOS. Chongqing: Chongqing University Press; 2010 (in Chinese).

22. Meng ZX. Study on Cross-Cultural Adjustment and Application of Frommelt Attitude Toward care of the Dying Patient. Beijing: Beijing University of Chinese Medicine; 2014 (in Chinese).

23. Gerdtz MF, Daniel C, Dearie V, Prematunga R, Bamert $M$, Duxbury J. The outcome of a rapid training program on nurses' attitudes regarding the prevention of aggression in emergency departments: a multi-site evaluation. Int J Nurs Stud. 2013;50:1434-1445.

24. Soares $\mathrm{MH}$, de Vargas $\mathrm{D}$. The translation and cultural adaptation of the Management of Aggression and Violence Attitude Scale - MAVAS - for nurses in Brazil. Rev Esc Enferm USP. 2013;47:899-906 (in Portuguese).

25. Wan $\mathrm{CH}$. Quality of Life Measurement Method. Beijing: Beijing Medical University Press; 2000 (in Chinese). 